\title{
Organisatorische Komplexität in sozialen Bewegungen: Das Beispiel globaler Dschihadismus
}

\begin{abstract}
Andreas Streiter*
Zusammenfassung

Dieser Artikel illustriert die organisationstheoretisch relevanten Aspekte der von Gilles Deleuze und Félix Guattari entworfenen Rhizom-Metapher anhand des Fallbeispiels globaler Dschihadismus. Dabei werden zwei Ziele verfolgt: Erstens soll das Potenzial der Metapher aufgezeigt werden, zu einem besseren Verständnis dynamischer Organisationsmodalitäten beizutragen, die durch die Linse traditioneller Theorien betrachtet schwer zugänglich sind. Zweitens soll zu einem umfassenderen Verständnis des Phänomens globaler Dschihadismus beigetragen werden. Mithilfe der Rhizom-Metapher wird deutlich, dass der globale Dschihadismus nicht entweder hierarchisch oder netzwerkartig organisiert ist, sondern dass er eine organisatorische Vielheit darstellt, die hierarchische und netzwerkartige Dimensionen in sich ständig verändernden Ausprägungen umfasst.
\end{abstract}

Schlagwörter: Organisation, Rhizom, Dschihadismus, al-Qaeda, IS

\section{Global Jihadism and Organizational Complexity in Social Movements}

\section{Abstract}

This article illustrates the "rhizome" metaphor of Gilles Deleuze and Félix Guattari with the example of global jihadism. The aim of the article is twofold: first, it shows the potential of the metaphor to contribute to a better understanding of dynamic modalities of organizing. Second, it contributes to a more comprehensive understanding of global jihadism as an empirical phenomenon. By implementing the rhizome metaphor, it becomes apparent that the organization of global jihadism extends beyond an either/or duality of hierarchy and network, but that it is an organizational multiplicity which comprises both hierarchical and network dimensions at the same time. The extent of those dimensions is constantly changing.

Keywords: Organization, Rhizome, Jihadism, al-Qaeda, IS

*Andreas Streiter, Universität Innsbruck.E-Mail: andreas.streiter@uibk.ac.at 


\section{Einleitung}

Der globale Dschihadismus ist wohl eine der machtvollsten sozialen Bewegungen unserer Zeit. Kaum eine andere Bewegung hat es geschafft, die Lebenswelt von Millionen, wenn nicht Milliarden von Menschen weltweit in nur wenigen Jahren so fundamental zu verändern; dass diese Veränderungen alles andere als positiv sind, tut dem Phänomen als interessantem Forschungsfall keinen Abbruch. Der Erfolg der Bewegung hat viele Gründe, ein Teilaspekt ist die extrem effektive Kombination und Gleichzeitigkeit von verschiedenen, auf den ersten Blick unvereinbar erscheinenden Organisationsmodalitäten. Mit dem vorliegenden Beitrag wird anhand des Konzepts des „Rhizoms“ der Philosophen Gilles Deleuze und Félix Guattari eine neue Sichtweise auf die Organisationsmodalitäten des globalen Dschihadismus eröffnet und somit zu einem besseren Verständnis des Phänomens beigetragen. Gleichzeitig wird das Konzept des Rhizoms anhand des Fallbeispiels erklärt und sein Potenzial aufgezeigt, ein erweitertes Verständnis von dynamischen Organisationsmodalitäten zu entwickeln, welche durch die Linse traditioneller Organisationstheorien betrachtet nur schwer zugänglich sind.

Der Bedarf nach neuen Konzepten zum besseren Verständnis der Organisationsmodalitäten des globalen Dschihadismus wird sowohl in der Fachliteratur als auch im medialen Diskurs deutlich: Zum einen herrscht seit längerer Zeit ein Akademikerstreit darüber, ob der globale Dschihadismus primär hierarchisch oder netzwerkartig organisiert ist (siehe Sageman 2004, 2008; Sageman/Hoffman 2008; Hoffman 2008). Zum anderen ist in neuester Zeit eine Debatte darüber entbrannt, wie das weit verbreitete Konzept des „Lone-Wolf-Terroristen“ zu bewerten ist (Burke 2017; Gartenstein-Ross 2017; Easterly/Geltzer 2017; Gartenstein-Ross/Barr 2016). Diese Debatte dreht sich vor allem darum, dass auch Terroristen, die keine augenscheinlichen Verbindungen zu einer Terrororganisation haben, nicht Teil eines klassischen Netzwerks sind und ihre Angriffe weitestgehend autonom ausführen, nicht in einem Vakuum operieren, sondern meist Verbindungen, wenn auch nur sehr lose oder virtuelle, zu Gleichgesinnten haben. Traditionelle Konzepte wie hierarchische Organisationen, aber auch Netzwerke können diese Gleichzeitigkeit autonomen Handelns und ideologischer Einbettung in eine globale Bewegung nur unzureichend erfassen.
Das Konzept des Rhizoms kann uns sowohl dabei helfen, die Gleichzeitigkeit von hierarchischen und netzwerkartigen Organisationsmodalitäten im globalen Dschihadismus zu erfassen, als auch zu einem besseren Verständnis von dschihadistischen Akteuren beizutragen, die weder Teil einer hierarchischen Organisation noch eines Netzwerkes im engeren Sinne sind. Des Weiteren kann es unsere Aufmerksamkeit darauf lenken, dass es für eine nachhaltige Bekämpfung des globalen Dschihadismus notwendig ist, das Phänomen als Ganzes zu erfassen, anstatt sich auf (organisatorische) Teilaspekte zu konzentrieren. Im verbleibenden Teil der Arbeit wird zuerst der globale Dschihadismus definiert und seine Konzeption als soziale Bewegung diskutiert, danach das Konzept des Rhizoms erklärt und mit Fallbeispielen des globalen Dschihadismus illustriert. In der Diskussion werden die theoretischen, praktischen und politischen Konsequenzen aufgezeigt, welche ein rhizomatisches Verständnis des globalen Dschihadismus zur Folge haben.

\section{Was ist globaler Dschihadismus?}

Im medialen und akademischen Diskurs existieren eine Reihe von Begriffen, die ein und dasselbe Phänomen bezeichnen: „globaler Dschihadismus“, „globaler salafistischer Dschihad“, oder, weniger präzise, aber nicht weniger verbreitet „internationaler islamischer/ islamistischer Terrorismus“. Marc Sageman (er verwendet die Bezeichnung "globaler salafistischer Dschihad") beschreibt das Phänomen kompakt und präzise:

„The Global Salafi Jihad is a worldwide religious revivalist movement with the goal of reestablishing past Muslim glory in a great Islamist state stretching from Morocco to the Philippines, eliminating present national boundaries. It preaches salafiyyah (from salaf, the Arabic word for , ancient one and referring to the companions of the Prophet Mohammed), the restoration of authentic Islam, and advocates a strategy of violent jihad, resulting in an explosion of terror to wipe out what it regards as local political heresy. The global version of this movement advocates the defeat of the Western powers that prevent the establishment of a true Islamist state." (Sageman 2004: 1)

Al-Qaeda und der selbsterklärte „Islamische Staat“ (IS) sind die zwei prominentesten Akteure innerhalb dieser Bewegung, aber bei Weitem nicht die einzigen. Es gibt zahlreiche kleinere Gruppierungen und Einzelpersonen weltweit, die sich der Bewegung zugehörig fühlen. Manche dieser Gruppierungen haben al-Qaeda oder dem IS „offiziell“ die Treue geschworen, andere 
haben lose Verbindungen zu al-Qaeda oder dem IS, wieder andere haben keine manifesten Verbindungen $\mathrm{zu}$ anderen bzw. bedeutenderen Gruppierungen. Was alle Akteure im globalen Dschihadismus eint, ist die oben beschriebene Ideologie und der Einsatz von Gewalt zur Erreichung ihrer Ziele. Gruppen mit einer ähnlichen Ideologie, die Gewalt ablehnen beziehungsweise gewalttätige Gruppen, die eine andere Ideologie und Ziele verfolgen (z.B. Hamas) können dem globalen Dschihadismus nicht zugerechnet werden.

\section{Globaler Dschihadismus: eine soziale Bewegung?}

Mit den Anschlägen vom 11. September trat der globale Dschihadismus ins Blickfeld der Weltöffentlichkeit. Kurz darauf entwickelte sich eine lebhafte Debatte unter AkademikerInnen, SicherheitsexpertInnen und PolitikgestalterInnen, wie das Phänomen am besten $\mathrm{zu}$ beschreiben sei. Während die ursprünglich vorherrschende Sichtweise davon ausging, dass al-Qaeda als hierarchische Organisation die zentrale Rolle bei den Anschlägen spielte und deshalb im Fokus des „War Against Terror" stehen sollte, setzte sich bald eine differenziertere und umfassendere Betrachtungsweise durch. Insbesondere Marc Sageman (2004, 2008) argumentierte, dass al-Qaeda als hierarchische Organisation im Hintergrund nur noch eine untergeordnete Rolle im globalen Dschihadismus spiele. Nach Sageman lässt sich das Phänomen am besten als soziale Bewegung beschreiben:

„It is not a specific organization, but a social movement consisting of a set of more or less formal organizations, linked in patterns of interaction ranging from the fairly centralized [...] to the more decentralized [...] and with various degrees of cooperation." (Sageman 2004: 137)

Wie Diani (1992) ausführt, gibt es keine allgemein akzeptierte Definition sozialer Bewegungen, allerdings teilen so gut wie alle gängigen Definitionen folgende drei Punkte:

„Social movements are defined as [1.] networks of informal interactions between a plurality of individuals, groups and/or organizations, [2.] engaged in political or cultural conflicts, [3.] on the basis of shared collective identities." (Diani 1992: 1)

Alle drei Punkte treffen auf den globalen Dschihadismus zu, sofern man ihn „als Ganzes“ betrachtet. Wie oben beschrieben, gibt es innerhalb der „Bewegung“ globaler Dschihadismus auch „Organisationen“, die zumindest im Kern hierarchisch organisiert sind. Dies ist für soziale Bewegungen nicht ungewöhnlich, das Konzept formal organisierter „Social Movement Orga- nizations (SMOs) “ ist in der Forschung seit Langem etabliert (McCarthy/Zald 1977; Zald/Ash 1966). Al-Qaeda und der IS können somit als SMOs innerhalb der breiteren sozialen Bewegung des globalen Dschihadismus konzeptualisiert werden. Wie oben erwähnt, sind diese Organisationen bei Weitem nicht die einzigen SMOs des globalen Dschihadismus, wenn auch die bedeutendsten. Wie unten beschrieben wird, ist die Grenze zwischen den SMOs und der breiteren Bewegung fließend; so ist die Entstehung des globalen Dschihadismus als Bewegung und Ideologie untrennbar mit der Entstehung al-Qaedas als hierarchischer Organisation verbunden. Des Weiteren ist beispielsweise die Frage von „Mitgliedschaft“ beim IS weit komplexer als in „klassischen“ SMOs.

Ein weiterer Punkt, der einer Klärung bedarf, ist die Anwendung von Gewalt, einschließlich terroristischer Taktiken in sozialen Bewegungen. Im vorliegenden Beitrag wird die insbesondere im Bereich der Terrorismusforschung verbreitete Position eingenommen, dass auch Bewegungen, die terroristische Taktiken zur Erreichung ihrer Ziele anwenden, als „soziale“ Bewegungen konzeptualisiert werden können (Sageman 2004; Singerman 2003). Im Feld der Bewegungsforschung ist diese Position umstritten (Bosi/Demetriou 2015). Letztendlich bringt uns diese Frage wieder zu der Definition von sozialen Bewegungen zurück, die wie oben erwähnt nicht abschließend geklärt ist.

Kurz: Ich nehme die Position ein, dass der globale Dschihadismus als soziale Bewegung bezeichnet werden kann. Al-Qaeda und der IS können als SMOs konzeptualisiert werden, die Grenzen zwischen diesen SMOs und der breiteren Bewegung sind allerdings fließend.

Die Metapher des Rhizoms kann uns dabei helfen, das Zusammenspiel und die Gleichzeitigkeit von hierarchischen und netzwerkartigen Dimensionen in sozialen Bewegungen besser zu verstehen. Der globale Dschihadismus bietet sich als Fallbeispiel an, da hier diese Gleichzeitigkeit der Dimensionen besonders deutlich ersichtlich wird (vgl. Flyvbjerg 2006: 229).

Im folgenden Abschnitt wird das Konzept des Rhizoms vorgestellt. In weiterer Folge wird das Konzept anhand des Fallbeispiels globaler Dschihadismus näher erläutert.

\section{Rhizom als Metapher}

Eines vorweg: Beim Rhizom handelt es sich um keine „Theorie“ im engeren Sinne. Im allgemeinen Sprachgebrauch handelt es sich bei einer Theorie um ein „System wissenschaftlich begründeter Aussagen zur 
Erklärung bestimmter Tatsachen od. Erscheinungen u. der ihnen zugrunde liegenden Gesetzlichkeiten" (Duden 2003: 1577, meine Hervorhebung). Wie unten beschrieben, stellen Deleuze und Guattari grundsätzlich in Frage, dass (sozialen) Phänomenen „Gesetzlichkeiten zugrunde liegen“, weshalb sie dem Anspruch, Gesetzmäßigkeiten herauszufinden, nicht gerecht werden können. Das „Rhizom“ ist daher als Metapher, nicht als Theorie zu verstehen. Wie von Morgan (1980) ausgeführt, sind Metaphern eine wichtige Voraussetzung für Theoriebildung - mithilfe neuer Metaphern können neue Theorien entwickelt werden, die Teilaspekte der Realität erklären können, welche mit traditionellen Metaphern unzugänglich sind. Sowohl bei Deleuze und Guattari als auch im vorliegenden Beitrag geht es somit weniger darum, operationalisierbare oder präskriptive Erkenntnisse zu gewinnen, als darum, eine neue Sichtweise auf Phänomene zu entwickeln und Dimensionen auszuleuchten, die mithilfe von traditionellen Theorien im Dunkeln bleiben. Um diese Erkenntnisse „verwertbar“ zu machen, bedarf es wiederum neuer Theoriebildung.

Wie die meisten Ideen von Deleuze und Guattari ist auch das „Rhizom“ ein sehr umfassendes, und daher relativ allgemeines Konzept, das Implikationen in den verschiedensten (Wissenschafts-) Bereichen hat. Für Deleuze und Guattari ist die Welt ein ständiges „Werden“ (im Gegensatz zum statischen „Sein“). Um dieses (offene) Werden zu erfassen, entwickeln sie das Konzept des Rhizoms, welches sie der Baummetapher gegenüberstellen. Diese grundlegende Ontologie des Werdens stellt auch den bedeutendsten Unterschied zu anderen, auf den ersten Blick ähnlichen Organisationstheorien dar. So entwickeln Ahrne/Brunsson (2009) das Konzept der "partiellen Organisation“, das wiederum von Den Hond et al. (2015) für die Bewegungsforschung aufgegriffen wird. Auch dieses Konzept befasst sich mit dem Nebeneinander von Modalitäten des Organisierens, konkret mit (formalen) Organisationen, Institutionen und Netzwerken. Der Unterschied zum Konzept des Rhizoms besteht vor allem darin, dass in der partiellen Organisation diese Organisationsmodalitäten bewusst getrennt werden. Dies steigert zwar die Analysekraft in Bezug auf die einzelnen Elemente, allerdings werden dadurch die Zusammenhänge zwischen diesen Organisationsmodalitäten in den Hintergrund gedrängt. In den Worten von Den Hond et al.: „by breaking down the concept of organization in its constituting elements $[\ldots]$, the notion of partial organization offers social movement scholars the language and conceptual tools to analyze and understand how ,organization' matters to social movements" (Den Hond et al. 2015: 300). Institutionen, Netzwerke und formale Organisationen werden somit als relativ statische Elemente innerhalb von sozialen Bewegungen gesehen, die sich im Laufe der Zeit zwar verändern können, aber dennoch unabhängig voneinander betrachtet und analysiert werden können und sollten.

Durch die Linse des Rhizoms betrachtet, passiert genau das Gegenteil: Die Trennung der einzelnen Organisationsmodalitäten rückt in den Hintergrund, stattdessen liegt der Fokus hier auf der Untrennbarkeit und der gegenseitigen Beeinflussung dieser Elemente. Soziale Bewegungen werden als Vielheit verstanden: „Eine Vielheit hat weder Subjekt noch Objekt; sie wird ausschließlich durch Determinierungen, Größen und Dimensionen definiert, die nicht wachsen, ohne dass sie sich dabei gleichzeitig verändert" (Deleuze/Guattari 1977: 13). Organisationen, Netzwerke und Institutionen werden hier als epistemologische Konstrukte verstanden, die dabei helfen, sich in dieser komplexen Vielheit besser zurechtzufinden, nicht als objektive, materielle Entitäten. Dies hängt wiederum mit der „Ontologie des Werdens“ zusammen, die dem Denken von Deleuze und Guattari zugrunde liegt. Chia (1999) nennt diese Ontologie zwar „metaphysics of change“, fasst das Konzept aber gut zusammen:

"[it] acknowledges the existence of an external fluxing reality, but denies our ability to accurately represent such a reality using established symbols, concepts and categories precisely because reality is ever-changing and hence resistant to description in terms of fixed categories. All representational attempts, according to this view, are forms of human abstraction emanating from our will to order. Representations do not simply correspond to reality. Rather they are simplifying devices which enable us to deal with what would otherwise be an intractable reality indifferent to our causes. Such a metaphysical position, therefore, accepts ontological realism, but rejects epistemological realism in favour of constructivism/social constructionism." (Chia 1999: 210)

Für das Verständnis des globalen Dschihadismus hat dies weitreichende Folgen: Wie unten genauer ausgeführt, lag der Fokus von Analysten, Sicherheitsorganen und Medien unmittelbar nach den Anschlägen vom 11. September auf formalen Terrororganisationen und deren Zerstörung, was den militärischen „War on Terror“ zur Folge hatte. Als die Infrastruktur und die hierarchische, formale Organisation al-Qaedas wei- 
testgehend zerstört waren, traten die netzwerkartigen Dimensionen des globalen Dschihadismus verstärkt in den Vordergrund. Sicherheitsbehörden und Analysten reagierten, Netzwerke und soziale Bewegungen lösten die formale Organisation als vorherrschende Konzeption des globalen Dschihadismus ab. In neuester Zeit rückte der Fokus immer mehr auf das Phänomen des „Lone-Wolf“-Terroristen, Einzeltäter ohne Verbindungen zu hierarchischen Organisationen oder Netzwerken. Dieser Fokus auf einzelne (organisatorische) Elemente hatte und hat zur Folge, dass andere Elemente ignoriert oder zumindest vernachlässigt werden, was wiederum zu problematischen und kontraproduktiven politischen Entscheidungen führen kann. Die RhizomMetapher kann uns dabei helfen, dieses Denken in Kategorien zu überwinden, da sie unser Augenmerk darauf lenkt, dass Netzwerke oder formale Organisationen eben nur menschliche Konstrukte sind, die uns helfen, die extrem komplexe und sich ständig im Wandel befindende Realität (des globalen Dschihadismus) besser zu verstehen.

Dabei ist es wichtig festzuhalten, dass ein rhizomatisches Verständnis des globalen Dschihadismus keinesfalls als Ersatz für traditionellere Theorien oder Perspektiven vorgeschlagen wird. Vielmehr kann das Rhizom eine wertvolle Ergänzung dieser Theorien sein, die uns hilft, formale Organisation und Netzwerke im Zusammenhang zu verstehen. Dieses zusammenhängende Verständnis hindert uns nicht daran, Teilaspekte des Gefüges mithilfe etablierter Theorien zu analysieren; allerdings kann mithilfe der Rhizom-Metapher ein Bewusstsein dafür geschaffen werden, dass ein einseitiger Fokus auf eines dieser Teilelemente zur Folge haben kann, dass andere Elemente, und vor allem die Verbindungen, Zusammenhänge und gegenseitigen Beeinflussungen zwischen diesen Elementen, vernachlässigt werden.

Im Folgenden wird das Konzept umrissen und anschließend anhand des Fallbeispiels die organisationstheoretisch relevanten Aspekte näher beleuchtet.

\section{Rhizom und Baumform}

Deleuze und Guattari entlehnten den Begriff des Rhizoms aus der Botanik, wo er eine meist unterirdisch wachsende Sprossachse bezeichnet. Rhizome können an jeder beliebigen Stelle geteilt werden, und aus allen Stücken des Rhizoms können sich neue Pflanzen entwickeln. Dies unterscheidet sie von Pflanzen, welche kein Rhizom bilden: Werden die Wurzeln eines Baums abgeschnitten, entwickelt sich eben kein neuer Baum aus den abgeschnittenen Wurzeln. Beispiele für rhizombildende Pflanzen sind Bambus, Efeu, oder Maiglöckchen. Der Begriff wird von Deleuze und Guattari verwendet, um eine Alternative zum im westlichen Denken vorherrschenden „Baum-Modell“ zu schaffen (Deleuze/Guattari 1977: 29-31). Das BaumModell kann in unserem Kontext am besten mithilfe eines Organigramms veranschaulicht werden: Es bildet Hierarchien und formale Organisationsstrukturen ab, aber es kann niemals die ganze Komplexität organisationaler Realität abbilden. Wie Rosenstiehl und Petitot ausführen, hat das Primat der Baumform nicht nur Auswirkungen auf unsere Wahrnehmung sozialer und organisatorischer Realität, sondern auch auf deren Konstruktion:

„accepting the primacy of hierarchical structures amounts to giving arborescent structures privileged status ... The arborescent form admits of topological explanation .... In a hierarchical system, an individual has only one active neighbor, his or her hierarchical superior .... The channels of transmission are preestablished: the arborescent system preexists the individual, who is integrated into it at an allotted place." (Rosenstiehl/Petitot 1974, zitiert in Deleuze/Guattari 2007: 16)

Das Konzept des Rhizoms steht der Baumform diametral gegenüber. Die Grundidee des Rhizoms wird von Deleuze und Guattari wie folgt umrissen:

"Als unterirdischer Sproß (sic) unterscheidet sich ein Rhizom grundsätzlich von großen und kleinen Wurzeln. Knollen und Knötchen sind Rhizome. Pflanzen mit großen oder kleinen Wurzeln können in vielerlei Hinsicht rhizomorph sein [...] Das Rhizom selbst kann die verschiedensten Formen annehmen, von der Verästelung und Ausbreitung nach allen Richtungen an der Oberfläche bis zur Verdichtung in Knollen und Knötchen. "(Deleuze) Guattari 1977: 11)

Deleuze und Guattari nennen folgende Merkmale des Rhizoms; die einzelnen Prinzipien werden später anhand des Fallbeispiels näher erläutert.

„1. und 2. - Prinzip der Konnexion und der Heterogenität. Jeder beliebige Punkt eines Rhizoms kann und muß (sic) mit jedem anderen verbunden werden. Ganz anders dagegen der Baum oder die Wurzel, wo ein Punkt und eine Ordnung festgesetzt werden. "(Deleuze/Guattari 1977: 11)

In dieser Passage wird vor allem das Prinzip der Konnexion verdeutlicht. Die Heterogenität ist eng verbunden mit dem nächsten Prinzip, dem Prinzip der Vielheit. Heterogenität bedeutet, dass Dinge oder Konzepte, welche normalerweise als Entität wahrge- 
nommen werden, in Wirklichkeit aus der Konnexion von verschiedenen Elementen entstehen, die bei einem im Baum-Modell verhafteten Denken als andere Entitäten wahrgenommen werden, welche mit dem eigentlichen Betrachtungsgegenstand nichts oder nur wenig zu tun haben. Deleuze und Guattari verdeutlichen dies mit einer Kritik an Chomskys $(1956,1959)$ Linguistik. Deleuze und Guattari führen aus, dass Sprache eben nicht eine Entität darstellt, die ausschließlich mit „objektiven“ grammatikalischen Gesetzmäßigkeiten erklärt werden kann, sondern dass unzählige Elemente wie etwa „Machtorganisationen, Ereignisse in Kunst, Wissenschaft und gesellschaftlichen Kämpfen“ (Deleuze/Guattari 1977: 12) einen Einfluss auf die Entstehung und unser Verständnis von Sprache haben. Im Gegensatz zu Chomsky gehen Deleuze und Guattari davon aus, dass Sprache nicht aus sich selbst heraus verstanden und analysiert werden kann, sondern dass sie eine Konnexion von heterogenen Elementen darstellt, die mitgedacht werden müssen: „Die Sprache mit einer rhizomatischen Methode analysieren heißt [...], sie auf andere Dimensionen und andere Register [als die der Sprachstruktur selbst] hin zu dezentrieren. Nur als Funktion von Entmachtung zieht sich eine Sprache auf sich selbst zurück“" (Deleuze/Guattari 1977: 13).

„3. - Prinzip der Vielheit: nur wenn das Viele als Substantiv, als Vielheit behandelt wird, hat es keine Beziehung mehr zum Einen als Subjekt und Objekt, als Natur und Geist, als Bild und Welt. [...] Eine Vielheit hat weder Subjekt noch Objekt; sie wird ausschließlich durch Determinierungen, Größen und Dimensionen definiert, die nicht wachsen, ohne dass sie sich dabei gleichzeitig verändert." (Deleuze/Guattari 1977: 13)

Sehr verkürzt dargestellt ist das Prinzip der Vielheit eine Alternative zur Unterscheidung zwischen dem „Einen“ und dem „Vielen“, zwischen „Subjekt“ und „Objekt“. Vielheiten befinden sich in einem ständigen Prozess des Entstehens, Wandels und Vergehens. Man kann von einer Vielheit nichts entfernen oder hinzufügen, ohne die Vielheit als Ganzes zu verändern. Vielleicht wird das Konzept der Vielheit am besten durch den ersten Satz von Deleuze und Guattaris „Tausend Plateaus" verdeutlicht:

"The two of us wrote Anti-Oedipus [Prequel von ,Tausend Plateaus'] together. Since each of us was several, there was already quite a crowd. Here we have made use of everything that came within range, what was closest as well as farthest away." (Deleuze/Guattari 2007: 3)

Indem sie sich zu einer „Menge“ zusammentaten, formten sie schon eine Vielheit, die Gebrauch von allem machte, was in ihre Reichweite kam. Aus dieser Vielheit wurde ein Buch, das Menschen und Gedanken beeinflusst, welche wiederum Teil der Vielheit werden. Mit dem Verfassen dieses Aufsatzes werde ich Teil der Vielheit und verändere sie zugleich, da Deleuze und Guattari sicher nicht geplant hatten, dass ihre Ideen zur Analyse des globalen Dschihadismus verwendet werden. Es ist noch wichtig zu erwähnen, dass Deleuze und Guattari nicht der Ausgangspunkt dieser Vielheit waren, da eine Vielheit keinen Ausgangspunkt hat. Bei der Vielheit geht es um Verbindungen und Dimensionen, nicht um Punkte. Die Gedanken von Deleuze und Guattari wurden von anderen Gedanken, anderen Vielheiten beeinflusst und indem sie ein Buch zusammen verfassten, veränderten sie diese Vielheiten und wurden zugleich Teil davon.

„4. - Prinzip des asignifikanten Bruchs: gegen die übersignifikanten Einschnitte, die die Strukturen voneinander trennen oder eine davon durchqueren. Ein Rhizom kann an jeder beliebigen Stelle gebrochen und zerstört werden; es wuchert entlang seiner eigenen oder anderen Linien weiter. Man wird mit den Ameisen nicht fertig, weil sie ein tierisches Rhizom bilden: es rekonstruiert sich auch dann noch, wenn es schon größtenteils zerstört ist." (Deleuze/Guattari 1977: 16)

Dieses Prinzip ist relativ verständlich formuliert und benötigt daher an dieser Stelle keine weitere Erklärung; es wird im weiteren Verlauf des Artikels mit Fallbeispielen aus dem globalen Dschihadismus illustriert.

„5. und 6. - Prinzip der Kartographie und der Dekalkomonie (sic) ${ }^{1}$ : ein Rhizom ist keinem strukturalen oder generativen Modell verpflichtet. Es kennt keine genetischen Achsen oder Tiefenstrukturen." (Deleuze/Guattari 1977: 20)

Deleuze und Guattari wollen damit aussagen, dass ein Rhizom im Gegensatz zu einer Baumform niemals kopiert werden kann. In einer Baumform sind immer die gleichen Punkte miteinander verknüpft, es können beliebig viele Kopien erstellt werden, die alle exakt gleich aussehen (z.B. Organigramm). Ein Rhizom hingegen verändert sich ständig, es kann somit nur kartographiert werden. Der Unterschied zu einer Kopie besteht darin, dass eine Kopie vorgibt, ein Abbild der Realität darzustellen. Eine Karte hingegen stellt diesen Anspruch nicht. Sie kann allerdings Verbindungen in einem Rhizom abbilden, oder, präziser, konstruieren.

1 Décalcomanie $=$ künstlerische Technik des Farbabzuges; Verfahren, Abziehbilder herzustellen. 
Eine Karte bildet Realität nicht ab, sie ist Teil der Konstruktion von Realität.

Im nächsten Teil der Arbeit werden diese doch recht sperrigen Prinzipien anhand der historischen Entwicklung des globalen Dschihadismus veranschaulicht.

\section{Die Entstehung des globalen Dschihadismus: ein rhizomatischer Prozess des Werdens}

Obwohl seine ideologischen Wurzeln Jahrhunderte zurückverfolgt werden können, handelt es sich beim globalen Dschihadismus um ein relativ neues Phänomen. Die Ideologie entwickelte sich während der sowjetischen Invasion in Afghanistan in den späten 1980er-Jahren. Während der Invasion strömten ausländische Kämpfer aus verschiedensten Ländern nach Afghanistan, um die afghanischen Mujaheddin in ihrem Kampf gegen die Sowjets zu unterstützen. Saudische Salafisten kamen so in Kontakt mit radikalen politischen ägyptischen Gruppierungen wie „al-Jihad“ (Wiktorowicz 2006: 225). Die Vermengung der radikalen politischen Ideen der Ägypter mit dem salafistischen Glauben der Saudis führte zur Entstehung einer neuen Ideologie, des "salafistischen Dschihadismus“ (Kepel 2003: 96-97).

$\mathrm{Zu}$ diesem Zeitpunkt hatte der salafistische Dschihadismus allerdings noch keine globale Ausrichtung. Das ursprüngliche Ziel der meisten ausländischen Kämpfer beschränkte sich darauf, Afghanistan von den Sowjets zu befreien und die Errichtung eines islamischen Staates zu unterstützen. Im Laufe der Zeit entwickelte sich allerdings ein neuer „Frame“ (Snow et al. 1986; Tarrow 1998): Der Kampf in Afghanistan wurde zunehmend als Teil eines größeren, weltweiten Befreiungskampfes der Muslime wahrgenommen. Al-Qaeda spielte bei der Entstehung dieses Frames eine zentrale Rolle. Die Organisation wurde 1988 von Osama bin Laden, der zu diesem Zeitpunkt bereits eine Schlüsselrolle in der Organisation der ausländischen Kämpfer in Afghanistan innehatte, zusammen mit einigen Weggefährten gegründet. Nachdem sich der Abzug der sowjetischen Truppen abzeichnete, hatten die ausländischen Kämpfer ihr ursprüngliches Ziel erreicht; viele konnten oder wollten allerdings nicht in ihre Heimatländer zurückkehren. Unter dem Einfluss des Ägypters Ayman al-Zawahiri, der später die Nummer zwei al-Qaedas werden sollte und nach dem Tod Osama bin Ladens die Führung der Organisation übernahm, entwickelten Osama bin Laden und einige seiner Anhänger die Idee, in Afghanistan eine „Basis“ („al-Qaeda“ auf Arabisch) zu etablieren, von der aus ein weltweiter Dschihad zur Verbreitung eines Islams salafistischer Prägung geführt werden sollte (Bergen 2006).

Hier werden die Prinzipien 1, 2 und 3 (Konnexion, Heterogenität und Vielheit) zum ersten Mal ersichtlich: die Entstehung al-Qaedas und damit des globalen Dschihadismus war ein rhizomatischer Prozess des Werdens: „Becoming indicates a process of symbiosis, the connection of heterogenous elements into new assemblages with emergent properties" (Chesters/ Welsh 2005: 188). Diese Verbindung von heterogenen Elementen zu neuen Gefügen mit neuen Eigenschaften und Bedeutungen, welche über die Summe der Teile des Gefüges hinausgehen, ist bei der Entstehung des globalen Dschihadismus klar erkennbar. Die Ideologie entwickelte sich mit der Verbindung von drei Hauptelementen: ägyptische Dschihadisten (deren vorrangiges Ziel es war, das ägyptische Regime zu stürzen), saudische Salafisten (die den vorwiegend politischen Kämpfen lokaler Dschihadisten eine neue religiöse Bedeutung gaben) und ausländische Kämpfer (die ursprünglich Afghanistan von der sowjetischen Besatzung befreien wollten und nach der Erreichung dieses Ziels nach einem neuen Sinn suchten). Erst die Verbindung dieser Elemente konnte den globalen Dschihadismus in der Form schaffen, die wir heute kennen. ${ }^{2}$

\section{Al-Qaeda zwischen hierarchischer Organisation und rhizomatischem Netzwerk}

Der Kern al-Qaedas war von Anfang an hierarchisch organisiert. Jamal al Fadl, einer der ersten Rekruten al-Qaedas, beschreibt die grundlegende Struktur der Organisation in den Anfangstagen folgendermaßen:

„At that time our general emir [is] Osama bin Laden.

Under the emir it's something called shura (consultative) council. Under shura council we have different committee. We have committee for military purpose. The emir of the military committee, his name Abu Ubaidah al Banjshiri. We got money and business committee. Under the money committee, we got office for immigration stuff. Like if

2 Natürlich spielten noch unzählige weitere Elemente, wie externe Einflüsse und Finanzen, eine Rolle; manche davon werden im weiteren Verlauf des Artikels behandelt. Gemäß dem „Prinzip der Kartographie“ bitte ich die LeserInnen im Hinterkopf zu behalten, dass auch dieser Artikel nur eine „Karte“ des globalen Dschihadismus zeichnen kann und daher gezwungenermaßen vereinfacht und nicht alle Elemente der Vielheit behandelt. 
you want to travel. We got another committee for media reporting and the newspaper." (Bergen 2006: 86-87)

Die formale Struktur al-Qaedas blieb in den folgenden Jahren weitestgehend unverändert. ${ }^{3}$ Neben dem hierarchischen Kern von al-Qaeda gab es allerdings immer auch eine rhizomatische Dimension, die vor allem in der Art der Planung und Ausführung der Anschläge auf westliche Ziele deutlich wird. Von Anfang an verfolgte al-Qaeda ein Prinzip der „Zentralisierung der Entscheidung und Dezentralisierung der Ausführung", welches von Osama bin Ladens ehemaligem Bodyguard Abu Jandal anhand des Anschlags auf das US-Kriegsschiff USS Cole folgendermaßen beschrieben wird:

„Al Qaeda pursues a method or principle that calls

for, centralization of decision and decentralization of execution. The decision was made centrally, but the method of attack and execution was the duty of field commanders. [...] The planning for the Cole operation was carried out by the people [on the ground]. The idea was formed and the target was set and then it was referred to a higher military control committee in al Qaeda called the Military Affairs Committee, which does not plan, but gives the green light, the support, and the funds for these operations. But, the planning, execution, and method of attack were all undertaken by field commanders in the operations field." (Bergen 2006: 253)

Die Initiative, die Planung und die Ausführung des Anschlages kamen von außerhalb der Hierarchie al-Qaedas: Die Operation wurde von Abdal Rahim al-Nashiri geplant. Obwohl er noch während der Planungsphase Mitglied von al-Qaeda wurde, war dem, als er die Operation Osama bin Laden vorschlug, nicht so. Die Idee wurde von außen an Osama bin Laden herangetragen, er gab lediglich "grünes Licht“ und unterstützte die Operation finanziell. Auch die Ausführung und Planung der Operation wurde von al-Nashiri weitestgehend autonom durchgeführt.

Die Anschläge vom 11. September waren auf die gleiche Weise organisiert: Idee und Initiative kamen von Khalid Sheikh Mohammed (KSM), der zum damaligen Zeitpunkt kein Mitglied al-Qaedas war. Die Ausführung des Planes erfolgte durch Zellen, welche von den Organisatoren untereinander vernetzt wurden. Ramzi Binalshibh, neben KSM und Mohammed Atta einer der wichtigsten Organisatoren der Anschläge,

3 Für eine genauere Beschreibung der formalen Struktur al-Qaedas und der handelnden Personen siehe Gunaratna/Oreg (2010). erklärte die Vorgangsweise in einem Interview mit $\mathrm{Al}$ Jazeera folgendermaßen:

"As for your question about the coordination [of the 9/11 attacks], it is simply a process of interconnecting various cells, establishing a line of contact between these cells and the General Command in Afghanistan as well as following up on work priorities of these cells until all phases of preparation are complete up to the moment of execution." (Bergen 2006: 303-304)

Obwohl al-Qaeda im Kern hierarchisch organisiert war, waren zwei der prominentesten Anschläge also eher Bottom-up- als Top-down-Operationen. Die Initiativen kamen von Personen, die vor den Anschlägen nur vage Verbindungen zur Organisation hatten. Auch die Planung und Ausführung wurden weitestgehend autonom vom hierarchischen Kern durchgeführt, dessen Rolle sich auf die Autorisierung, Finanzierung und zu einem gewissen Grad auf die Verbindung der beteiligten Akteure beschränkte.

Al-Qaeda kann in der Phase vor 9/11 also als hierarchische Organisation beschrieben werden, die gleichzeitig signifikante rhizomatische Dimensionen hatte. Nach 9/11 verschob sich das Verhältnis dieser zwei Dimensionen: Die rhizomatischen Dimensionen gewannen enorm an Bedeutung, während die hierarchischen Dimensionen an Bedeutung verloren. Erst durch das Erstarken der rhizomatischen Dimensionen konnte sich der globale Dschihadismus von einer Ideologie mit einer sehr überschaubaren Anhängerzahl zu einer globalen Bewegung entwickeln.

\section{Al-Qaeda und globaler Dschihadismus nach 9/11: mehr Rhizom, weniger Hierarchie}

Nach den Anschlägen vom 11. September griff eine US-geführte Koalition Afghanistan an, entfernte die Taliban von der Macht und setzte eine US-freundliche Regierung ein. Die Trainingscamps und sonstige Infrastruktur al-Qaedas wurden zerstört, ein Großteil der Führungsriege gefangen genommen oder getötet. Die verbleibenden Kader flohen größtenteils ins afghanisch-pakistanische Grenzgebiet, ständig auf der Flucht vor Drohnen und Spezialkommandos. Für jede klassische, hierarchische Organisation hätte solch eine Situation wohl das Aus bedeutet, wie beschrieben hatte al-Qaeda neben ihrer hierarchischen allerdings auch bedeutende rhizomatische Dimensionen. Diese wurden durch die Angriffe der Koalitionstruppen nicht nur nicht beeinträchtigt, im Gegenteil: Durch die mediale Aufmerksamkeit wurde der Zulauf zum glo- 
balen Dschihadismus ungemein verstärkt. Während alQaeda und Osama bin Laden zuvor relativ unbekannt waren, unterstützten die Angriffe auf Afghanistan das Narrativ al-Qaedas, dass der Westen einen Krieg gegen die muslimische Welt führe, und der globale Dschihadismus entwickelte eine zuvor unvorstellbare Anziehungskraft für potenzielle Rekruten. Ein Interview mit Sami ul Haq, einem einflussreichen pakistanischen Kleriker und flüchtigem Bekannten Osama bin Ladens, kurz nach den ersten Luftschlägen gegen al-Qaeda 1998 illustriert diese Situation; man kann davon ausgehen, dass die Angriffe gegen Afghanistan 2001 ein Vielfaches der Mobilisierungswirkung der Luftschläge von 1998 hatten:

„I think America has made Osama a supernatural being. Wherever the terrorism occurs, right away they think of him. I don't think he has such influence, or such control and resources. Osama bin Laden has become a symbol for the whole Islamic world. All those outside powers who are trying to crush Muslims interfering with them; he is the courageous one who raised his voice against them. Yes, he is a hero to us, but it is America itself who first made him a hero." (Bergen 2006: 228)

Die weitgehende Zerstörung der hierarchischen Kernorganisation zusammen mit der neuen Bekanntheit hatte entscheidende Konsequenzen für die Organisationsmodalitäten al-Qaedas: Wie Hoffman (2006) ausführt, transformierte sich al-Qaeda in den Jahren nach 9/11 von einer prinzipiell hierarchischen Organisation (wenngleich mit signifikanten rhizomatischen Dimensionen) in eine netzwerkartige, vorwiegend rhizomatisch organisierte Bewegung: „a vast enterprise - an international franchise with like-minded local representatives, loosely connected to a central ideological or motivational base, but advancing the remaining center's goals at once simultaneously and independently of each other" (Hoffman 2006: 3). Innerhalb dieser Bewegung können nach Hoffman (2006) vier Teilbereiche identifiziert werden:

1. Al-Qaeda Central: Die verbliebene, immer noch hierarchisch organisierte Führungsriege; hält sich vorwiegend in und um Pakistan auf.

2. Al-Qaeda Affilliates and Associates: Formal organisierte dschihadistische Gruppen, welche im Laufe der Jahre von al-Qaeda Unterstützung erhielten und dadurch immer noch relativ eng mit al-Qaeda Central verbunden waren (z. B. al-Qaeda im Irak, aus der später der Islamische Staat hervorgehen sollte).

3. Al-Qaeda Locals: Gruppen und Individuen, die in der Vergangenheit Kontakte mit al-Qaeda Central hatten; im Unterschied zu den „Affilliates und Associates" sind/waren diese Kontakte aber weit weniger intensiv und in vielen Fällen rissen die Kontakte nach 9/11 komplett ab.

4. Al-Qaeda Network: „homegrown“ Dschihadisten, welche von al-Qaeda lediglich inspiriert wurden, aber trotz fehlender direkter Kontakte zu al-Qaeda oder einer anderen identifizierbaren dschihadistischen Organisation bereit sind, Anschläge zu verüben.

Hier werden Prinzipien 4, 3, und 2 des Rhizoms besonders deutlich ersichtlich: Das Prinzip des asignifikanten Bruches und die Prinzipien der Vielheit und Heterogenität. Al-Qaeda wurde nach der Militärintervention in Afghanistan nicht zerstört, vielmehr „wucherte“ die Bewegung weiter, wenn auch außerhalb der hierarchischen Strukturen von al-Qaeda Central. Es wurde zunehmend ersichtlich, dass die Konzeption von al-Qaeda als homogene Organisation nicht mehr akkurat war. Die meisten Akteure des globalen Dschihadismus, egal ob „offiziell“ mit al-Qaeda Central assoziierte Gruppen oder selbstradikalisierte Einzeltäter ohne greifbare Verbindungen zu al-Qaeda Central, bekannten sich zwar zu al-Qaeda, waren aber nicht in die hierarchischen Strukturen der Kernorganisation eingebunden. Aus diesem Grund gingen viele BeobachterInnen ab dieser Zeit dazu über, das Phänomen nicht mehr als „Organisation al-Qaeda“ sondern als „Bewegung globaler Dschihadismus“ zu konzeptualisieren (Sageman 2004, 2008; Gunaratna/Oreg 2015; Stewart 2014).

Die Grenzen zwischen der hierarchischen Organisation al-Qaeda und der breiteren Bewegung des globalen Dschihadismus verschwammen zusehends, was uns wiederum zu Prinzipien 5 und 6, „Kartographie und der Dekalkomonie“, bringt. Die sich ständig im Wandel befindlichen Dimensionen und Organisationsmodalitäten entzogen sich zunehmend den etablierten analytischen Kategorien, was an einem in der Terrorismusforschung prominenten Akademikerstreit zwischen Bruce Hoffman und Marc Sageman besonders deutlich wird: Hoffman vertrat die Ansicht, dass der globale Dschihadismus immer noch weitestgehend hierarchisch gesteuert war, beziehungsweise dass al-Qaeda Central weiterhin eine herausragende Rolle spielte, während Sageman argumentierte, dass der globale Dschihadismus praktisch ausschließlich netzwerkartig organisiert war. Hoffman und Sageman lieferten sich in mehreren Artikeln und Büchern eine hitzige Debatte über das Thema, welche bis heute nicht abschließend gelöst ist (Sageman 2004, 2008; Hoffman 
2008; Sageman/Hoffman 2008; Hoffman/Reinares 2014).

Sowohl Hoffmans Bild der hierarchischen Organisation als auch Sagemans Netzwerk können als „Kopien“ etablierter Konzepte verstanden werden. Wie von Deleuze und Guattari ausgeführt, lässt sich ein Rhizom allerdings nicht kopieren, sondern nur „kartographieren“. Wie oben beschrieben, besteht der Unterschied darin, dass eine Kopie vorgibt, ein exaktes Abbild der Realität darzustellen, während eine Karte lediglich Verbindungen in einem Rhizom konstruiert. Eine Karte kann uns helfen, Teilaspekte einer komplexen Realität zu verstehen und begreifbar zu machen. Sie stellt allerdings nicht den Anspruch, Realität so abzubilden „wie sie ist“. Mit der Metapher des Rhizoms lässt sich der Widerspruch zwischen hierarchischer Organisation und Netzwerk auflösen, weil anerkannt werden kann, dass der globale Dschihadismus eben nicht entweder hierarchisch oder netzwerkartig ist, sondern eine Vielheit, welche beide Dimensionen in sich ständig verändernden Ausprägungen umfasst. Sowohl hierarchische Organisation als auch Netzwerke werden in dieser Sichtweise als epistemologische Konstrukte gesehen, die uns helfen diese Vielheit zu verstehen und zugänglich zu machen, nicht als objektive Entitäten.

In den Jahren nach 9/11 kann der globale Dschihadismus also als Rhizom mit signifikanten hierarchischen Dimensionen gesehen werden, wobei der wichtigste hierarchische „Knoten“ innerhalb des Rhizoms immer noch al-Qaeda Central war. Dies änderte sich erst ab 2014.

\section{Der „Islamische Staat": ein neuer hierarchischer Knoten}

Mit dem kometenhaften Aufstieg des Islamischen Staates (IS) 2014 wurde eine neue hierarchische Organisation zur Speerspitze des globalen Dschihadismus. Die Organisation war unter wechselnden Namen und unter wechselnder Führung seit 2001 im Irak aktiv. Von 2004 bis 2014 war sie offiziell mit al-Qaeda assoziiert, bevor diese Verbindung aufgrund strategischer Differenzen von al-Qaeda (Central) für beendet erklärt wurde (Turner 2015). In den Jahren davor war die Organisation nach einigen Rückschlägen fast vollständig zerstört worden; vor allem in der Führungsriege entstand eine Lücke, nachdem 34 der 42 bekannten Anführer getötet oder gefangen genommen worden waren (Reuter 2015: 52). Das entstandene Machtvakuum wurde vorwiegend von ehemaligen Militär- und Geheimdienstoffizieren des Saddam-Hussein-Regimes gefüllt. Diese neue Führungsriege war zum Großteil nicht besonders religiös oder ideologisch, ihre Motivation war vor allem die Wiedererlangung politischer Macht. Unter der neuen Führung gelang es dem IS sehr schnell, im Irak und Syrien ein Territorium von der Größe Großbritanniens zu erobern, was die Anziehungskraft für internationale Rekruten enorm verstärkte.

Im Unterschied zu al-Qaeda, deren Traum von einem islamischen Staat, einem Kalifat, stets eine Utopie geblieben war, gab es nun eine Organisation, die diese Utopie in Realität verwandelt hatte. In seinen eroberten Gebieten baute der IS eine staatsförmige Bürokratie auf; die interne Organisation des IS ist extrem hierarchisch und bürokratisch, was eine entscheidende Rolle beim raschen militärischen Erfolg in Syrien und im Irak spielte (Reuter 2015).

Neben der hierarchischen Organisation in den Kerngebieten des IS gibt es allerdings auch hier wieder eine rhizomatische Dimension, die besonders außerhalb seines Kernterritoriums in zwei Aspekten ersichtlich wird: Zum einen in der Verbindung mit dschihadistischen Gruppen außerhalb Syriens und des Iraks, zum anderen in der Präsenz des IS im Westen. Wie schon al-Qaeda zuvor schmiedet der IS Allianzen mit dschihadistischen Gruppierungen weltweit, die organisatorisch mit der Kernorganisation nicht oder nur lose verbunden sind und vorwiegend in begrenzten Regionen ihrer Herkunftsländer aktiv sind. ${ }^{4}$ Die Verbindung mit dem IS erfolgt durch einen Treueschwur an den „Kalifen“ Abu Bakr al-Baghdadi. Obwohl diese Gruppen also vorwiegend außerhalb der hierarchischen Strukturen des IS operieren und weiterhin vorwiegend ihre eigenen, (lokalen) Interessen verfolgen, sind sie doch „offiziell“ Teil des IS, wenn auch vorwiegend auf einer symbolischen Ebene.

Wieder werden die Prinzipien 1 (Konnexion), 2 (Heterogenität) und 3 (Vielheit) ersichtlich: Durch die Verbindung mit unterschiedlichen versprengten, lokalen Gruppen baut der IS seine Macht außerhalb seines Kernterritoriums aus. Diese Gruppen werden nun Teil der Vielheit „Islamischer Staat“. Dadurch verändern sich sowohl die Gruppen selbst als auch der Islamische Staat: Die vormals lokal agierenden Gruppen werden Teil eines globalen Projekts, und der Islamische Staat

4 Z.B. verschiedene Bürgerkriegsparteien in Libyen (US Department of State 2016) oder Boko Haram in Nigeria (Zeit Online 2015). 
wird in lokalen Konflikten aktiv, die weit außerhalb seines Kernterritoriums liegen, was wiederum das Narrativ des „weltweiten Kalifats“ und somit die Anziehungskraft für Sympathisanten und potenzielle RekrutenInnen verstärkt.

Hier wird in Ansätzen auch eine Organisationsmodalität erkennbar, die bei den Aktivitäten des IS im Westen noch stärker deutlich wird: IS ist, wer sich zum IS erklärt. Während ausländische KämpferInnen im Kernterritorium des IS bürokratisch erfasst und einem Platz in der strikten Hierarchie zugewiesen werden, sind die Zutrittshürden im Westen weniger hoch bzw. nicht existent. Die Anschläge im Westen erfolgen prinzipiell nach zwei Mustern: Erstens gab es eine Reihe von Anschlägen, welche wie die Anschläge al-Qaedas nach dem oben beschriebenen Prinzip „Zentralisierung der Entscheidung und Dezentralisierung der Ausführung“ organisiert waren. Beispiele hierfür sind die Anschläge von Paris am 13. November 2015 oder die Brüsseler Anschläge am 22. März 2016 (Callimachi 2016). Zumindest bis 2015 war der Großteil der Anschläge im Westen allerdings anders geartet: Hier gab es keinerlei Verbindungen zur Hierarchie des IS, die Täter wurden aber von der Propaganda des IS inspiriert und bekannten sich zur Organisation (Hegghammer/Nesser 2015). Der IS nimmt diese Geschenke dankend an und reklamiert die Anschläge für sich. Ein Großteil der Medien springt auf diesen Zug auf und macht aus isolierten, zum Teil psychisch beeinträchtigten Einzeltätern IS-Mitglieder. Wie Musharbash (2016) ausführt, sind dies zum Großteil Menschen, „die vermutlich mehr Persönlichkeitsmerkmale mit Amokläufern ohne extremistischen Hintergrund teilen als mit jenen islamistischen Attentätern, die wir bisher kennen“. Sympathisanten ohne jegliche Verbindungen zur Kernorganisation zu terroristischen Anschlägen zu ermuntern, ist ein bewusster Teil der Strategie des IS, wie ein Aufruf des offiziellen Sprechers der Organisation, Abu Muhammad al-Adnani, vom September 2014 illustriert:

"If you can kill a disbelieving American or European - especially the spiteful and filthy French - or an Australian, or a Canadian, or any other disbeliever from the disbelievers waging war, including the citizens of the countries that entered into a coalition against the Islamic State, then rely upon Allah, and kill him in any manner or way however it may be. Do not ask for anyone's advice and do not seek anyone's verdict. Kill the disbeliever whether he is civilian or military, for they have the same ruling. "(Hegghammer/Nesser 2015)
Beispiele für diese Art von Anschlägen sind die Anschläge von Kopenhagen am 14. und 15. Februar 2015 oder der Anschlag auf einen Nachtclub in Orlando am 12. Juni 2016. Hier liegt ein wichtiger Unterschied zu al-Qaeda: die Organisation begrüßte zwar vereinzelt Anschläge, die von Nicht-al-Qaeda-Mitgliedern verübt wurden, allerdings nahm sie solche Anschläge nicht für sich in Anspruch und erklärte sie nicht im Nachhinein zu al-Qaeda-Operationen (Mendelsohn 2016). Die Zugangshürden, um ein „offizielles“ Mitglied al-Qaedas zu werden, sind sehr hoch. Anders beim IS: Im Prinzip kann jeder, der sich zum IS bekennt ein „offizielles“ Mitglied der Organisation werden. Obwohl der IS im Kern also extrem hierarchisch organisiert ist, versucht die Führungsriege (im Gegensatz zu al-Qaeda) nicht, die rhizomatischen Dimensionen zu kontrollieren oder „einzufangen“. Im Gegenteil: Der IS ist sich der Stärke seiner „unkontrollierten“ rhizomatischen Dimensionen völlig bewusst und versucht diese, durch Aufrufe wie den obigen, gezielt zu verstärken. Hier besteht auch ein wichtiger Unterschied zu Netzwerken: Viele dieser Einzeltäter sind nicht Teil eines klassischen Netzwerks. Wie Sageman (2004: 135) ausführt, sind in einem Netzwerk enge soziale Beziehungen der entscheidende Faktor, sich dem globalen Dschihadismus anzuschließen: „Social bonds are the critical element in this process and precede ideological commitment. These bonds facilitate the process of joining the jihad through mutual emotional and social support, development of a common identity and encouragement to adopt a new faith." Wie beschrieben haben viele Einzeltäter keine engen Beziehungen zu einem „echten“ Netzwerk; andererseits operieren sie auch nicht in einem Vakuum, sondern haben, meist online, lose und diffuse Verbindungen zu Gleichgesinnten: „Islamic militants [...] emerge as often from the fertile and desperately depressing world of online jihadism - with its execution videos, mythologised history, selectively read religious texts and Photoshopped pictures of alleged atrocities against Muslims - as from organised groups that meet in person" (Burke 2017). Diese Dschihadisten sind weder Teil einer hierarchischen Organisation, noch Teil eines Netzwerks. Trotzdem sind sie ein (zunehmend an Bedeutung gewinnender) Teil der rhizomatischen Vielheit des globalen Dschihadismus.

\section{Diskussion und Fazit}

Der globale Dschihadismus war von Anfang an eine organisatorische Vielheit. Die hierarchische Organi- 
sation „al-Qaeda“ entwickelte sich ab Ende der achtziger Jahre des vergangenen Jahrhunderts parallel zum breiteren Phänomen des globalen Dschihadismus. Die Entstehung der hierarchischen und der rhizomatischen Dimensionen war dabei eng verknüpft - die eine wäre ohne die andere nicht möglich gewesen. Ohne die Kombination von hierarchischen und rhizomatischen Organisationsmodalitäten hätte sich der globale Dschihadismus auch niemals zu der machtvollen Bewegung entwickelt, mit der wir heute konfrontiert sind.

Die rhizomatischen und die hierarchischen Dimensionen innerhalb des globalen Dschihadismus waren immer vorhanden, wenn auch in unterschiedlichen Ausprägungen. Während bis 9/11 die hierarchische Kernorganisation al-Qaedas bei praktisch allen dschihadistischen Anschlägen auf westliche Ziele eine entscheidende Rolle spielte (auch wenn die Operationen immer auch rhizomatische Charakteristika aufwiesen), verschoben sich die Dimensionen nach der weitestgehenden Zerstörung der Infrastruktur alQaedas in Afghanistan. Die immer schon vorhandenen rhizomatischen Dimensionen gewannen enorm an Bedeutung, trotzdem existierte auch der hierarchisch organisierte Kern al-Qaedas weiter und spielte eine signifikante Rolle bei Anschlägen im Westen.

Mit dem Erstarken des Islamischen Staats ab 2014 verlor al-Qaeda an Bedeutung, der IS entwickelte sich zum wichtigsten hierarchischen Knoten innerhalb des globalen Dschihadismus. Neben der Eroberung eines großen Territoriums in Syrien und im Irak (was ohne eine hierarchische Organisation nicht möglich gewesen wäre), lässt sich der Aufstieg des IS durch die bewusste Kultivierung seiner rhizomatischen Dimensionen erklären: Sowohl durch die Verbindung mit lokalen dschihadistischen Gruppen, welche durch einen Treueschwur an Abu-Bakr al Baghdadi sehr einfach zu einem Teil des IS werden können, ohne in die formale Hierarchie eingebettet zu sein, als auch durch die Vereinnahmung von EinzeltäterInnen im Westen. Hierin besteht auch ein signifikanter Unterschied $\mathrm{zu}$ al-Qaeda: Während sich al-Qaeda als Elite des globalen Dschihadismus sah, sieht sich der IS selbst als Massenbewegung, bei der jede/r Mitglied werden kann, sofern er oder sie bereit ist, im Namen des IS Anschläge zu verüben; der Führung von al-Qaeda war und ist es wichtig, einen gewissen Grad an Kontrolle auch über die rhizomatischen Dimensionen der Organisation zu behalten; die Führung des IS hat keinerlei Probleme damit, wenn diese Dimensionen außerhalb ihrer Kontrolle „wuchern“.
Angesichts der Komplexität und der ständigen Wandlung von Organisationsmodalitäten des globalen Dschihadismus stoßen traditionelle Konzepte und Metaphern von „Organisation“ an ihre Grenzen. Die meisten etablierten Organisationstheorien fußen implizit oder explizit auf solch traditionellen Konzepten und Metaphern. Die vorherrschenden Analysekategorien sind hier statische Organisationen; Wandel und Veränderung werden nachrangig behandelt. Zwar trug die Rekonzeptualisierung des globalen Dschihadismus als soziale Bewegung (im Gegensatz zum zuvor gängigen Bild der hierarchischen Organisation) viel zu einem besseren Verständnis des Phänomens bei, allerdings werden auch traditionelle Theorien zu sozialen Bewegungen der Dynamik und Komplexität des Phänomens nicht vollständig gerecht. So ist beispielsweise die Trennung zwischen "Social Movements" und „Social Movement Organizations" für die Analyse von herkömmlichen, vorwiegend in nationalen Kontexten operierenden sozialen Bewegungen zweifellos geeignet. Bei globalen, höchst dynamischen Phänomenen wie dem globalen Dschihadismus greifen solche statischen, „trennenden“ Konzeptionen allerdings zu kurz (Chesters/Welsh 2005: 189-190). Wie in dieser Arbeit gezeigt wurde, kann zwischen dem globalen Dschihadismus als Bewegung und hierarchischen Organisationen innerhalb dieser Bewegung, wie al-Qaeda oder dem IS, nicht strikt getrennt werden. Es handelt sich hier um verschiedene Dimensionen derselben Vielheit, die sich ständig im Wandel befinden und gegenseitig beeinflussen. Den globalen Dschihadismus durch die Linsen traditioneller Theorien zu sozialen Bewegungen zu betrachten, macht das Phänomen zwar einfacher fassbar, seine Dynamik und Komplexität wird dadurch allerdings verschleiert. Die Metapher des Rhizoms kann uns dabei helfen, neue Theorien zu entwickeln, welche der Komplexität und dem ständigen Wandel von (organisatorischen) Phänomenen wie dem globalen Dschihadismus besser gerecht werden.

Obwohl die Theoriebildung hier noch am Anfang steht, gibt es bereits bedeutende Ansätze in diese Richtung: Im Bereich der sozialen Bewegungen wäre ein Beispiel hierfür Meluccis (1995; 1996) Fokus auf die Bedeutung von prozessualer kollektiver Identitätskonstruktion für kollektives Handeln oder Chesters/ Welshs (2005) Betrachtung des „Alter-Globalization Movements“. Auch in der „allgemeinen“ Organisationsforschung wurde das Konzept des Rhizoms bereits von mehreren Autoren aufgegriffen (Chia 1999; Kornberger et al. 2006; Carter/Jackson 2004); auch hier wird das Rhizom als Metapher verwendet, eine Theoriebildung 
im engeren Sinne steht noch aus. Trotzdem, oder vielleicht gerade deswegen, kann das Konzept des Rhizoms eine neue Sichtweise auf Organisationsmodalitäten eröffnen, welche durch die Linsen traditioneller Theorien betrachtet im Dunkeln bleiben würden, und so zu einem erweiterten Verständnis sozialer Bewegungen beitragen.

Wie schon in der Einleitung erwähnt, hat eine rhizomatische Betrachtungsweise des globalen Dschihadismus auch praktische und politische Implikationen: Es wird dadurch klarer, dass sich der globale Dschihadismus eben nicht durch die Zerstörung von hierarchischen Organisationen, das Ausheben von Netzwerken oder eine stärkere Überwachung von potenziellen Einzeltätern besiegen lassen wird. Um dem globalen Dschihadismus nachhaltig entgegenzutreten, müsste das Substrat des Rhizoms ausgetrocknet werden. Dieses Substrat ist wiederum eine Vielheit aus mannigfaltigen Elementen. Soziale, geopolitische, wirtschaftliche, ideologische, religiöse und kulturelle Faktoren spielen ebenso eine Rolle wie lokale Konflikte. Aufgrund dieser Komplexität gibt es keinen einfachen Weg das Substrat auszutrocknen; der globale Dschihadismus wird uns noch eine lange Zeit begleiten.

Somit verschiebt sich die Fragestellung von „Wie können wir den globalen Dschihadismus besiegen?“ hin zu „Wie können wir mit dem globalen Dschihadismus umgehen und ihn zumindest eindämmen?"

Hier gibt es eine Reihe von Maßnahmen, die nützlich sein können: verstärkte Jugendsozialarbeit, Bildungsinitiativen, interreligiöser Dialog, verstärkte Integrationsbemühungen, Entwicklungszusammenarbeit, Stärkung von zivilgesellschaftlichen Initiativen in autoritären Staaten etc. Solche Maßnahmen mögen langfristig den Zulauf zum globalen Dschihadismus verringern, allerdings werden sie kurzfristig keine Anschläge verhindern. Hierzu sind weiter polizeiliche und geheimdienstliche Maßnahmen nötig. Allerdings müssen wir uns als Gesellschaft klar werden, wie viele demokratische Freiheiten und Rechte wir bereit sind aufzugeben, um einzelne Anschläge zu verhindern. Der Großteil der massiven Einschränkungen von Bürgerrechten nach 9/11 wurde von der Bevölkerung hauptsächlich wegen des unrealistischen Versprechens akzeptiert, der globale Dschihadismus könne in absehbarer Zeit besiegt werden (Richardson 2007). Eine rhizomatische Perspektive auf den globalen Dschihadismus macht einmal mehr klar, dass dies eine Illusion ist und kann einen Impuls liefern, das Verhältnis von Freiheiten, Rechten und Sicherheit neu zu überdenken.

\section{Literatur}

Ahrne, G./Brunsson, N. (2009): Organization outside organizations: The significance of partial organization. Organization, 18 (1), 83-104.

Bergen, P.L. (2006): The Osama bin Laden I know: An oral history of al-Qaeda's leader. New York: Free Press.

Bosi, L./Demetriou, C. (2015): Social Movements and Political Violence. In: Wright, J. D. (Hg.): International Encyclopedia of the Social \& Behavioral Sciences (Second Edition). Oxford: Elsevier, 430-436.

Burke, J. (2017): The myth of the ,lone wolf' terrorist. Guardian, 30.03.2017. Online: https://www.theguardian.com/ news $/ 2017 / \mathrm{mar} / 30 / \mathrm{myth}$-lone-wolf-terrorist [31.10.2017]

Callimachi, R. (2016): How ISIS Built the Machinery of Terror Under Europe's Gaze. Online: http://www.nytimes. com/2016/03/29/world/europe/isis-attacks-paris-brussels.html [03.08.2016].

Carter, P./Jackson, N. (2004): Gilles Deleuze and Felix Guattari. In: Linstead, S. (Hg.): Organization Theory and Postmodern Thought. London: Sage.

Chesters, G./Welsh, I. (2005): Complexity and Social Movement(s): Process and Emergence in Planetary Action Systems. Theory, Culture \& Society, 22 (5), 187-211.

Chia, R. (1999): A ,Rhizomic' Model of Organizational Change and Transformation: Perspective from a Metaphysics of Change. British Journal of Management, 10, 209-227.

Chomsky, N. (1956): Three models for the description of language. IRE Transactions on information theory, 2 (3), $113-124$.

Chomsky, N. (1959): On certain formal properties of grammars. Information and Control, 2 (2), 137-167.

Deleuze, G./Guattari, F. (1977): Rhizom. Berlin: Merve-Verlag.

Deleuze, G./Guattari, F. (2007): A thousand plateaus: Capitalism and schizophrenia. Minneapolis, Minn.: Univ. of Minnesota Press.

Den Hond, F./De Bakker/Frank G.A./Smith, N. (2015): Social Movements and Oragnizational Analysis. In: Della Porta, D./Diani, M. (Hg.): The Oxford Handbook of Social Movements. New York: Oxford University Press, Incorporated, 291-305.

Diani, M. (1992): The concept of social movement. The Sociological Review, 40 (1), 1-25.

Duden (Hg.) (2003): Duden - Deutsches Universalwörterbuch. Mannheim: Duden-Verlag.

Easterly, J./Geltzer, J.A. (2017): The Islamic State and the End of Lone-Wolf Terrorism. Online: http://foreignpolicy. $\mathrm{com} / 2017 / 05 / 23 /$ the-islamic-state-and-the-end-of-lonewolf-terrorism/ [18.07.2017].

Flyvbjerg, B. (2006): Five Misunderstandings About CaseStudy Research. Qualitative Inquiry, 12 (2), 219-245.

Gartenstein-Ross, D. (2017): Lone Wolves No More: The Decline of a Myth. Online: https://www.foreignaffairs.com/ 
articles/united-kingdom/2017-03-27/lone-wolves-nomore [18.07.2017].

Gartenstein-Ross, D./Barr, N. (2016): The Myth of Lone-Wolf Terrorism: The Attacks in Europe and Digital Extremism. Online: https://www.foreignaffairs.com/articles/ western-europe/2016-07-26/myth-lone-wolf-terrorism [18.07.2017].

Gunaratna, R./Oreg, A. (2010): Al Qaeda's Organizational Structure and its Evolution. Studies in Conflict \& Terrorism, 33 (12), 1043-1078.

Gunaratna, R./Oreg, A. (2015): The global Jihad movement: A handbook. Lanham: Rowman \& Littlefield.

Hegghammer, T./Nesser, P. (2015): Assessing the Islamic State's Commitment to Attacking the West. Perspectives on Terrorism, 9 (4), 14-30. Online: http://www.terrorismanalysts.com/pt/index.php/pot/article/view/440/871 [31.10.2017]

Hoffman, B. (2006): Combating Al Qaeda and the Militant Islamic Threat: Testimony presented to the House Armed Services Committee, Subcommittee on Terrorism, Unconventional Threats and Capabilities on February 16, 2006. Online: http://www.rand.org/pubs/testimonies/CT255. html [13.07.2016].

Hoffman, B. (2008): The Myth of Grass-Roots Terrorism: Why Osama bin Laden Still Matters. Foreign Affairs 87 (3), 133-138.

Hoffman, B./Reinares, F. (Hg.) (2014): The evolution of the global terrorist threat: From 9/11 to Osama bin Laden's death. New York: Columbia Univ. Press.

Kepel, G. (2003): The origins and development of the Jihadist movement: from anti-communism to terrorism. Asian Affairs, 34 (2), 91-108.

Kornberger, M./Rhodes, C./Bos, R.t. (2006): The Others of Hierarchy: Rhizomatics of Organising. In: Fuglsang, M./Sørensen, B. M. (Hg.): Deleuze and the social. Edinburgh: Edinburgh University Press.

McCarthy, J.D./Zald, M.N. (1977): Resource Mobilization and Social Movements: A Partial Theory. American Journal of Sociology, 82 (6), 1212-1241.

Melucci, A. (1995): The Process of Collective Identity. In: Johnston, H./Klandermans, B. (Hg.): Social Movements and Culture. University of Minnesota Press, 41-63.

Melucci, A. (1996): Challenging codes: Collective action in the information age. Cambridge [England], New York: Cambridge University Press.

Mendelsohn, B. (25.8.2016): ISIS' Lone-Wolf Strategy. Online: https://www.foreignaffairs.com/articles/2016-08-25/isislone-wolf-strategy [05.09.2016].

Morgan, G. (1980): Paradigms, Metaphors, and Puzzle Solving in Organization Theory. Administrative Science Quarterly, 25 (4), 605-622.

Musharbash, Y. (2016): Islamischer Staat: Aber er hat doch IS gesagt! Online: http://www.zeit.de/politik/ausland/2016-06/orlando-attentaeter-islamischer-staat-medien/komplettansicht [02.09.2016].
Reuter, C. (2015): Die schwarze Macht: Der „Islamische Staat“ und die Strategen des Terrors. München: Deutsche Verlags-Anstalt.

Richardson, L. (2007): What terrorists want: Understanding the enemy, containing the threat. New York: Random HouseTrade Paperbacks.

Sageman, M. (2004): Understanding terror networks. Philadelphia: University of Pennsylvania Press.

Sageman, M. (2008): Leaderless Jihad: Terror networks in the twenty-first century. Philadelphia, Pa.: Univ. of Pennsylvania Press.

Sageman, M./Hoffman, B. (2008): The Reality of Grass-Roots Terrorism [with Reply]. Foreign Affairs, 87 (4), 163-166.

Singerman, D. (2003): The Networked World of Islamist Social Movements. In: Wiktorowicz, Q. (Hg.): Islamic activism. A social movement theory approach. Bloomington, Ind.: Indiana Univ. Press, 143-163.

Snow, D.A./Rochford, E.B./Worden, S.K./Benford, R.D. (1986): Frame Alignment Processes, Micromobilization, and Movement Participation. American Sociological Review, 51 (4), 464-481.

Stewart, S. (2014): Jihadism in 2014: Defining the Movement. Online: https://www.stratfor.com/weekly/jihadism2014-defining-movement [11.11.2016].

Tarrow, S. (1998): Power in movement: Social movements and contentious politics. Cambridge: Cambridge Univ. Press.

Turner, J. (2015): Strategic differences: Al Qaeda's Split with the Islamic State of Iraq and al-Sham. Small Wars \& Insurgencies, 26 (2), 208-225.

US Department of State (2016): Terrorist Designations of ISIL-Yemen, ISIL-Saudi Arabia, and ISIL-Libya. Online: https://www.state.gov/j/ct/rls/other/des/266495.htm [31.10.2017]

Wiktorowicz, Q. (2006): Anatomy of the Salafi Movement. Studies in Conflict \& Terrorism, 29 (3), 207-239.

Zald, M.N./Ash, R. (1966): Social Movement Organizations: Growth, Decay and Change. Social Forces, 44 (3), 327341.

Zeit Online (13.3.2015): „Islamischer Staat“ droht mit Anschlägen im Westen. Online: http://www.zeit.de/politik/ ausland/2015-03/is-buendnis-boko-haram [31.10.2017] 